\title{
Challenges in Liver Transplantation
}

\author{
Chair: \\ Helge Bruns ${ }^{a} \quad$ Hans-Rudolf Raab ${ }^{a}$ \\ Participants: Kenya Yamanaka ${ }^{b}$ Kestutis Strupas $^{c}$ Peter Schemmer $^{d}$ \\ a University Department of General and Visceral Surgery, European Medical School Oldenburg-Groningen, Klinikum Oldenburg, \\ Oldenburg, Germany; \\ ${ }^{b}$ Department of Surgery, Kishiwada City Hospital, Osaka, Japan; \\ ${ }^{c}$ Center of Abdominal Surgery, Vilnius University Hospital Santariskiu Klinikos, Vilnius, Lithuania; \\ ${ }^{\mathrm{d} D e p a r t m e n t ~ o f ~ G e n e r a l, ~ V i s c e r a l ~ a n d ~ T r a n s p l a n t ~ S u r g e r y, ~ U n i v e r s i t y ~ H o s p i t a l ~ H e i d e l b e r g, ~ H e i d e l b e r g, ~ G e r m a n y ~}$
}

\begin{abstract}
Question 1: Organ shortage still is one of the major challenges in liver transplantation and ultimately leads to relevant waiting list mortality. How can waiting list mortality be decreased? How can the donor pool be increased? Would further prioritization of candidates on the waiting list be helpful or does this only postpone the existing problem?
\end{abstract}

Yamanaka: According to the Fact Book published by the Japanese Society for Transplantation in 2015 , the median waiting period of the 106 patients who underwent brain death liver transplantation in Japan from 2011 to 2014 was 377 days. The number of the candidates for liver transplantation in Japan is estimated to be around 2,200 patients every year. Thus the number of brain dead donors is absolutely insufficient. Therefore, prioritization of candidates on the waiting list is helpful, based on medical urgency. It is also helpful to consider how to make effective use of marginal donors. In Japan, where living-donor liver transplantation accounts for more than $90 \%$ of liver transplantations, the donor's intention is considered to be paramount, rather than the medical urgency.

Strupas: The National Transplant Bureau (NTB) is responsible for the increase in the number of donor organs in Lithuania. Seeking to reduce waiting list mortality, we prioritize patients waiting for liver transplantation according to the severity of their condition. Not only laboratory MELD (model for end-stage liver disease) scores are taken into the account. MELD exception points are added when certain diseases such as hepatocellular carcinoma (HCC), hepatopulmonary syndrome, portopulmonary syndrome and recurrent cholangitis persist. In order to reduce both waiting list and perioperative period mortality, the most consistent donor liver and a potential liver recipient couple is selected.
Schemmer: One major step in decreasing the organ shortage is to increase the general understanding of brain death (the definite death of a human being) and thus to increase the acceptance and willingness within our population to donate organs for transplantation.

Furthermore the graft function could be improved by graft reconditioning after donation supporting the general concept of decreasing graft injury during the process of organ donation which should be further developed, standardized, and clinically implemented. If those measures would still not increase the number of suitable organs donated for transplantation to meet the demand of grafts, living-donor liver transplantation of relatives could fill the remaining gap. This could include the concept of dual graft transplantation where two grafts are taken from two separate living donors and transplanted in one recipient to decrease the risk of smallfor-size graft syndrome (SFSS).

Further prioritization of candidates on the waiting list is merely a measure of shortage management.

\section{Question 2: Marginal organs and organs from extended criteria donors are frequently used to increase the donor pool but these livers may be more prone to primary dysfunction. Pharmacological and technical (e.g. machine perfusion) means aim to improve organ quality and to decrease reperfusion injury. What is the most promising approach?}

Yamanaka: A Japanese law does not permit to stop the life-support system; thus it is almost impossible to use a cardiac arrest donor for liver transplantation. In order to suppress ischemia/reperfusion injury (IRI) and SFSS, pharmacological treatments such as PDE III inhibitor (ex, olprinone) and glycine seem to be useful. In addition, establishment of cocktail treatment may be also useful to overcome the complexity of pathology of IRI and SFSS.

\section{KARGER}

(c) 2016 S. Karger GmbH, Freiburg

Fax +497614520714
Dr. med. Helge Bruns

University Department of General and Visceral Surgery

European Medical School Oldenburg-Groningen, Klinikum Oldenburg

Rahel-Strauss-Straße 10, 26133 Oldenburg, Germany

helge.bruns@ uni-oldenburg.de 
Strupas: Lithuania is a relatively small country with less than 3 million inhabitants. Donation is encouraged; however, its growth is slow. According to the NTB, among the EU countries Lithuania is ranked low place in terms of donation. Despite the contacts set between the Lithuanian NTB and Balttransplant, Scandiatransplant or Eurotransplant, we received only a few donor organs within the last 15 years (1 liver from Latvia, a few kidneys from Latvia and Estonia, a child's heart from France). That is why our approach and tactics towards marginal donor organs with a high probability of dysfunction is reserved. Marginal donor livers are used rarely. We had a practice, when the organ was used in an urgent case, but we could not receive another organ for re-transplantation, necessary due to the poor function of the first one.

Schemmer: There is a whole variety of beneficial concepts available, e.g., pharmacological and technical optimization of the graft quality in order to decrease the ischemic injury. Because of the multimodal mechanisms being responsible for graft injury prior, during and after transplantation, the preferable approach is the integration of both pharmacological and technical conditioning of the donor, the graft per se and the recipient. Machine perfusion can improve washout of injurious waste products, continuously supply oxygen and nutrients, and allow pharmaceutical interventions. Pharmacological conditioning is a practical strategy using a non-ischemic, non-hypoxic stimulus to protect against prolonged ischemia, increasing the graft survival even more in combination with machine perfusion.

\section{Question 3: Today, an arsenal of different immunosuppressive substances from different classes exists and allows both a renal sparing and individualized immunosuppression. What can we expect from novel immunosuppressive agents? How much individualization is possible? Is there a real place for induction of operational tolerance in the near future?}

Yamanaka: Though the short-term result of liver transplantation has become pretty well, the long-term prognosis was not much improved. For the long-term prognosis, humoral immunity is thought to be important. However, drugs that can suppress the humoral immunity effectively are not present. The idea of the prope tolerance which aims to use a dose of immunosuppressive drugs without side effects would be realistic. Measuring the donor-specific (DSA) and non-donor-specific antibody (non-DSA) may be useful for individualization of immunosuppression. It would be possible to control immunity prior to liver transplantation as induction therapy using a new antibody drug.

Strupas: Immunosuppression is individualized in our center. IL-2 receptor antibody (basiliximab) is used as an induction immunosuppression in about $30-40 \%$ of cases in order to preserve the renal function. Later on, lower doses of calcineurin inhibitors (CNIs) are prescribed. The main CNI used in our center is tacrolimus.
Schemmer: The modern immunosuppressive therapy consists of a combination of different kinds of immunosuppressive drugs, enabling lower dosages of the single drugs and thereby reducing the side effects. Individually tailored concepts based on the unique immune status of the patient are still under development.

To reduce the drug-associated complications of a CNI-based immunosuppression, induction therapy can be used. The induction therapy with antibodies against specific T cells of their receptors can delay the immunosuppressive therapy with CNI and decrease the risk of rejection at the same time.

The immunosuppressive therapy can be monitored with specific parameters like the blood level of the drug, the kidney and liver function, and immune system parameters. Based on these parameters, the dosage of the immunosuppressive drugs can be individually adjusted on a daily basis. Furthermore, the choice of a specific immunosuppressive regimen is based on previous diseases and the status of both the liver and kidney.

\section{Question 4: Transplantation needs teamwork and close interaction of specialists from various fields. How is this organized in your institution?}

Yamanaka: Pharmacists advised surgeons of the adjustment of the dose of an immunosuppressive agent or a specific antibiotic according to the blood concentration. Nutritionists are involved in nutritional management of patients before liver transplantation. Physical therapists perform rehabilitation of patient before surgery. The infection control team makes an advice for serious infection in the perioperative period of liver transplantation. However, surgeons do a lot of things rather than liver transplantation itself basically, if you look from the world standard.

Strupas: Liver transplantation for adults with terminal liver diseases or malignancies is based on a multidisciplinary, team-based approach. A multidisciplinary team of healthcare professionals, including gastroenterologists, nephrologists, transplantation surgeons, diagnostic radiologists, intensive care specialists, anesthesiologists, nurses, and interventional radiologists in our clinic have been trained in high-volume centers and know exactly what and when needs to be done in each new case. These specialists meet every month to review the patients' progress, ensure an individualized treatment, and reach a consensus on the next steps.

In 2004, when the liver transplantation program was launched, there were a few surgeons. For the time being, we have rotating surgical teams for explantation and implantation.

Schemmer: The interdisciplinary management of postoperative complications in liver transplantation is of extreme importance. Due to organ shortage and prioritization of the most severely ill recipients in the MELD-based allocation, both donor- and recipient-associated morbidity are increasing. An interdisciplinary, structured monitoring concept is essential for the timely identification and specific treatment of postoperative complications. Inter- 
disciplinary clinical rounds, laboratory testing, and Doppler ultrasound monitoring of the graft perfusion are as important as comprehensive anti-infection prophylaxis and immunosuppression. Arterial perfusion disorders of any kind, biliary complications, and postoperative fluid accumulation demand individualized therapeutic concepts. In summary, the success of liver transplantation depends on the communication and coordinated interdisciplinary cooperation of all disciplines involved.

\section{Question 5: Liver transplantation has evolved gradually with many small steps leading to decreased mortality and improved overall patient and organ survival. Some developments have been outstanding (e.g. the introduction of modern immunosuppressive drugs and the development of the split-liver technique). In your opinion, what single development has had the greatest impact in liver transplantation in the last decade?}

Yamanaka: The antibody-related analysis by Labscreen method has come to be performed in liver transplantation. Preoperative evaluation of donor liver using $\mathrm{MeV}$ is has become routine.

Strupas: The philosophy of our hospital is to provide liver transplantation services to all who may benefit, including those who are deemed to be of high risk or not considered for transplantation by other transplantation centers. Advanced immunosuppression has reduced the incidence of acute rejection, but has no effect on the incidence of chronic immune damage in any organ. Improved surgical technique, standardization of preservation techniques, and gentle organ manipulation surely reduce the ischemic injury and lead to better outcomes. But nowadays, the greatest impact in my opinion is a multidisciplinary team-based approach for patients' care.

Schemmer: The development of remote preconditioning lead to a novel strategy to reduce the IRI. The protective stimulus can be applied noninvasively where a blood pressure cuff is placed on a lower of upper limb to induce brief episodes of non-lethal IRI.

Furthermore, machine perfusion is particularly useful for the preservation and reconditioning of an organ obtained from extended criteria donors or donation after cardiac death and could expand the donor pool.

Further, one of the most important developments would be a tailored immunosuppression that is adjusted to the individual immune status of the patient, thus decreasing both complications and rejection rates at the same time.
Question 6: Milan Criteria, which are based on the imaging quality of the 1990s, are widely used to select patient with HCC for liver transplantation. Besides Milan Criteria, other criteria (e.g. University of San Francisco Criteria and 'up-to-seven' classification) have been explored. Can expansion of the criteria for HCC be justified? How should this be done?

Yamanaka: It would be preferable to expand the Milan criteria under a careful clinical trial. For example, the Kyoto University expands it, based on Kyoto Criteria. However, the expansion of the criteria should be done cautiously because of the shortage of donor liver.

Strupas: Lithuania has adopted the Milan Criteria for the prioritization of the waiting list for liver transplantation due to HCC. Besides, we perform 'bridge' surgery procedures for those who are awaiting a liver transplant (liver resection operations, radiofrequency-induced radiotherapy (RFITT), transarterial chemoembolization (TACE)).

Schemmer: In general an indication for liver transplantation should be given in patients who would benefit from transplantation with an increased survival compared to other treatment options. This would also be valid for palliation in HCC patients with tumors far outside Milan criteria provided that enough grafts would be available for all possible indications. However, this practice is currently not used because of both the dramatic organ shortage and the national regulations in Germany.

The dramatic shortage of donor organs leads to a moral dilemma when considering extending the selection criteria. Extended criteria may lead to denial of organs to patients who may benefit more from a liver transplantation, whereas not extending the criteria may lead to denying organs to patients who may benefit from transplantation in terms of prolonged survival.

\section{Participants}

\section{Dr. Helge Bruns}

University Department of General and Visceral Surgery

European Medical School Oldenburg-Groningen, Klinikum Oldenburg

Rahel-Strauss-Straße 10, 26133 Oldenburg, Germany

helge.bruns@uni-oldenburg.de

Prof. Dr. Hans-Rudolf Raab

University Department of General and Visceral Surgery

European Medical School Oldenburg-Groningen, Klinikum Oldenburg

Rahel-Strauss-Straße 10, 26133 Oldenburg, Germany

a-chirurgie@klinikum-oldenburg.de

Prof. Dr. Peter Schemmer

Department of General, Visceral and Transplant Surgery

University Hospital Heidelberg

Im Neuenheimer Feld 110, 69120 Heidelberg, Germany

Peter.Schemmer@med.uni-heidelberg.de 
Prof. Dr. Kestutis Strupas

Center of Abdominal Surgery

Vilnius University Hospital Santariskiu Klinikos

Santariskiu 2, 20021 Vilnius, Lithuania

kestutis.strupas@santa.lt
Dr. Kenya Yamanaka

Department of Surgery

Kishiwada City Hospital

1001, Gakuhara-cho, Kishiwada-shi, Osaka 596-8501, Japan

kenya-y@hotmail.co.jp 